\title{
Stepwise implementation of a cardiovascular risk management care program in primary care
}

\author{
Geert Smits ${ }^{1,2^{*}}$, on behalf of the PoZoB primary care group, Monika Hollander ${ }^{2}$, Sander van Doorn ${ }^{2}$ and
} Michiel Bots ${ }^{2}$

\begin{abstract}
Background: Primary care plays a pivotal role in sustainable cardiovascular risk management (CVRM) but little is known about the organizational process of implementing the guidelines. The aim of the study was to describe the approach taken by a primary care group to implement the CVRM guideline.
\end{abstract}

Methods: Stepwise introduction and implementation of a programmatic CVRM care program was organized and facilitated by the care group between April 2010 and January 2013 in 137 affiliated general practices with 188 general practitioners (GPs), in the vicinity of Eindhoven, Netherlands. Care group support comprised sufficient staff, support with data extraction based on ICPC and ATC codes and with identification of eligible patients by scrutinizing patient health records and adequate coding of disease conditions.

Results: Patient selection based on availability of structured information on ICPC codes and risk factor levels from the electronic health records, led to 38,675 eligible patients in 2013. December 2019, the CVRM program was still running in 151 practices with 51,416 patients receiving programmatic CVRM care. Linking problems between 8 different electronic health record systems and the multidisciplinary information system for integrated care delayed adequate data collection until the beginning of 2013.

Conclusion: Commitment of affiliated GPs, a structured approach with adequate coding of diagnoses and risk factors, central data registration and additional funding for sufficient staff support are important conditions for the introduction and implementation of successful and sustainable programmatic CVRM care. This approach constitutes the basis for long-term follow up and annual evaluation.

Keywords: Primary care, General practice, Cardiovascular disease, Programmatic care, Practice nurse, Implementation

\section{Background}

Cardiovascular diseases (CVD) are the most important cause of morbidity and mortality in low, middle and high income countries $[1,2]$. The most important modifiable risk factors for CVD are smoking, unhealthy lifestyle (alcohol consumption, unhealthy diet, physical inactivity), overweight, elevated blood pressure, unfavorable lipid levels and diabetes mellitus. These are considered

*Correspondence: g.smits@pozob.nl

${ }^{1}$ Bolwerk 11-14 5509, MH, Veldhoven, The Netherlands

Full list of author information is available at the end of the article to be responsible for $80-90 \%$ of preventable CVD [3]. National and international guidelines have been published to recommend approaches for prevention [4-6]. According to the Prevention Guideline of the European Society of Cardiology the general practitioner (GP) has a unique role in identifying individuals at risk for CVD and assessing their eligibility for intervention based on their risk profile" [7].

In the Netherlands many GPs are joint together in local or regional 'care groups'. A care group is an organization with a legal entity in which health care providers work together. The care group is responsible for the 
coordination and provision of contracted care in a particular region [8]. Care groups negotiate with regional health insurers about a bundled payment contract/integral funding. By joining a care group, practices benefit from a negotiated renumeration, support with implementation and regular education for health care providers. Care groups also organize chronic care programs for several conditions, such as diabetes mellitus type 2 (DM2), asthma/COPD (A/C) and cardiovascular risk management (CVRM). These chronic care programs are performed by a registered nurse with an additional training, in close collaboration with a GP. The Primary Care group PoZoB (Praktijk ondersteuning Zuid-oost Brabant) originated from an initiative of 6 smaller GP collectives, to which many other GPs voluntarily joined. PoZoB started with the implementation of chronic care programs for DM2 and A/C in 2005 and 2008 respectively, followed by CVRM in 2010. The content of the CVRM care program was based on the 2006 CVRM guidelines of the Dutch Society of General Practice [4]. The guideline provided the GP with recommendations on who to screen but made no recommendations on how to organize screening. Furthermore, the effect of strict application of the guideline in terms of cardiovascular risk control and prevention of vascular events in general practice had not been evaluated. This provided the 'research gap' at the start of the study. To the best of our knowledge, we are among the first describing the introduction and stepwise implementation of a carefully designed cardiovascular risk management program offered by a primary care group.

\section{Methods}

\section{Study population}

At the start of the CVRM implementation project in 2010, the care group comprised 137 GP practices (188 GPs) with 402,623 registered patients living in the southeastern part of the province of Brabant, and the western part of the province of Limburg in The Netherlands.

\section{Conditions for starting}

Before starting the implementation process, the PoZoB primary care group contacted every individual practice to identify suitability for participation in the CVRM care program, defined as i) participation in running chronic care programs for DM2 and $\mathrm{A} / \mathrm{C}$, and ii) having sufficient hours deployed for the practice nurse (PN) and the GP. The number of hours needed was estimated by the care group based on the number of participating patients, the number of annual consultations and the consultation time. If these conditions were met, the implementation process could start. Implementation start was linked to quarterly renumeration, therefore practices started every 3 months. The implementation start of 137 practices (188 GPs) quarterly is given in Table 1.

\section{Study design}

We designed the study as a non-experimental prospective cohort study embedded in routine clinical practice.

\section{Implementation steps that were taken}

The stepwise implementation comprised 4 steps: 1) Data extraction; 2) Inclusion/exclusion of eligible patients; 3) Consultation; 4) Follow up.

\section{1) Data extraction.}

Data extraction occurred from the patient electronic health record (EHR) to identify an increased risk for cardiovascular disease or with a history of cardiovascular disease. Extraction of data was carried out by a certified organization for multidisciplinary data management and scientific research ("Meetpunt Kwaliteit", since 2015 $\mathrm{INZO}=$ Instituut voor ZorgOptimalisatie). Potential eligibility for the program was based on medical diagnosis, and/or prescribed medication in patients older than 18 years and/or on labels that practices already used for identifying patients. Medical diagnosis was linked to the International Classification of Primary Care (ICPC) code and prescribed medication was linked to the Anatomical Therapeutical Chemical (ATC) classification. The ICPC and ATC codes used for identification are listed in Supplementary Table 1.

\section{2) Inclusion/exclusion of eligible patients.}

The PN plays an essential role in step 2, 3 and 4 of the implementation process. For in- and exclusion, the EHR was scrutinized by the $\mathrm{PN}$ if diagnoses or risk factors

Table 1 Practices and GPs starting implementation programmatic CVRM care

\begin{tabular}{|c|c|c|c|c|c|c|c|c|c|c|c|}
\hline \multirow{2}{*}{$\begin{array}{l}\text { Year } \\
\text { Quarter }\end{array}$} & \multicolumn{3}{|c|}{2010} & \multicolumn{4}{|c|}{2011} & \multicolumn{4}{|c|}{2012} \\
\hline & Q2 & Q3 & Q4 & Q1 & Q2 & Q3 & Q4 & Q1 & Q2 & Q3 & Q4 \\
\hline Practices (n) & 26 & 0 & 16 & 15 & 26 & 24 & 20 & 4 & 2 & 2 & 2 \\
\hline GPs (n) & 41 & 0 & 19 & 16 & 35 & 29 & 32 & 7 & 3 & 4 & 2 \\
\hline
\end{tabular}


were set correct, based on discharge letters from the specialist. Practices were given 1 year to complete the EHR investigation for correct ICPC codes and labeling, invite patients and start with CVRM care. A mandatory group course was offered to the GP and PN to standardize registration of patients. During this year, all practices were visited two or three times by specialized care group nurses to monitor progress and to discuss unclear diagnoses. If necessary, staff nurses were reachable by phone to discuss in- or exclusion in the CVRM care program.

Criteria for inclusion and exclusion in the CVRM care program were: (1) Patients with CVD or kidney disease: (i) documented ischemic or atherosclerotic heart disease (myocardial infarction and angina pectoris), heart failure, atrial fibrillation, aneurysm of the abdominal aorta, peripheral arterial disease, transient ischemic attack, ischemic or hemorrhagic stroke, chronic kidney disease and (ii) primarily treated in primary care and (iii) aged 18 years or above. (2) Patients without a history of CVD or kidney disease but at high risk of CVD (i) a 10 year cardiovascular mortality risk $>5 \%$, based on the SCORE table from the 2006 CVRM guidelines of the Dutch Society of General Practice (4) or (ii) use of blood pressure lowering or lipid lowering drugs in men aged $\geq 55$ years and women aged $\geq 60$ years or (iii) Systolic blood pressure $>180 \mathrm{mmHg}$ and/or total cholesterol $>8 \mathrm{mmol} / \mathrm{l}$ ever measured, independent of the 10 year mortality risk. Furthermore, these patients needed to be primarily treated in primary care and aged 18 years or above.

Exclusion criteria for both groups were: (i) primarily treated by a specialist in a hospital or at an outpatient clinic or (ii) diabetes mellitus (as they received cardiovascular risk management in a diabetes care program). Criteria are based on the CVRM guidelines of the Dutch Society of General Practice [4].

Those eligible were flagged in the EHR using the Care $2 U$ system. In 2010 a Multidisciplinary Information System for integrated care (Care2U) was introduced. Care2U data registered by the PN ended up automatically in the EHR. All patients were labeled in Care2U and in the EHR as follows:

- V1: Patients at high risk for CVD, treatment and follow up by the GP/PN

- V2: Patients at high risk for CVD, treatment and follow up by a specialist in hospital or out-patient clinic

- V3: Patients at high risk for CVD, refusing care by a GP/PN

- Z1: Patients with CVD, treatment and follow up by the GP/PN

- Z2: Patients with CVD, treatment and follow up by a specialist in hospital or out-patient clinic

- Z3: Patients with CVD, refusing care by a specialist
Ultimately, only patients who had their treatment and follow up in primary care (V1 and Z1) were included in the CVRM care program.

\section{3) Consultation}

Once a patient was eligible for CVRM care he/she received an invitation letter from the general practice to make an appointment with the PN. If the patient did not respond within 2 weeks, the practice assistant called the patient. During the first consultation the patient was asked whether he/she was willing to participate in the CVRM care program. Time for the intake was estimated $45 \mathrm{~min}$ and was often split in 2 visits to the practice. The intake comprised an interview, check of in- and exclusion criteria, a physical examination and referral for blood testing to the local laboratory to complete the cardiovascular risk profile. The main items from the interview the PN conducted with the patient are summarized in Table 2.

The protocolized physical examination, based on the CVRM guidelines of the Dutch Society of General Practice, assessed blood pressure, height and weight and body mass index $\left(\mathrm{BMI} \mathrm{kg} / \mathrm{m}^{2}\right)$, heart rate and waist circumference. Blood and urine samples were taken for fasting glucose, lipids (total cholesterol, HDL, LDL and triglycerides) and kidney function (serum creatinine, estimated glomerular filtration ratio (eGFR) and proteinuria). Blood tests performed less than 3 months ago could also be used. Blood sampling was performed at a local hospital laboratory or at a local diagnostic health center. Based on test results, for patients without a history of a cardiovascular disease and without preventive cardiovascular medication, a 10 year cardiovascular risk was estimated using the Systematic Coronary Risk Evaluation (SCORE) table from the 2006 CVRM guidelines of the Dutch Society of General Practice (4). For patients younger than 55 (men) or 60 (women) years of age the SCORE had to be calculated based on blood pressure or cholesterol levels before starting medication. If the SCORE was $\leq 5 \%$, the patient was not eligible for the CVRM care program.

\section{4) Treatment and follow-up}

After determining the cardiovascular risk profile, the PN made an individual care plan with the patient and supported self-management by informing about CVD or risk factors and motivating the patient to take the lead in coping with it in the best possible way. The PN discussed the individual care plan with the GP, who had final medical responsibility. The PN was responsible for preparing and discussing the individual care plan with the patient, comprising non-pharmacological 
Table 2 Summary of the aspects addressed during PNs interview

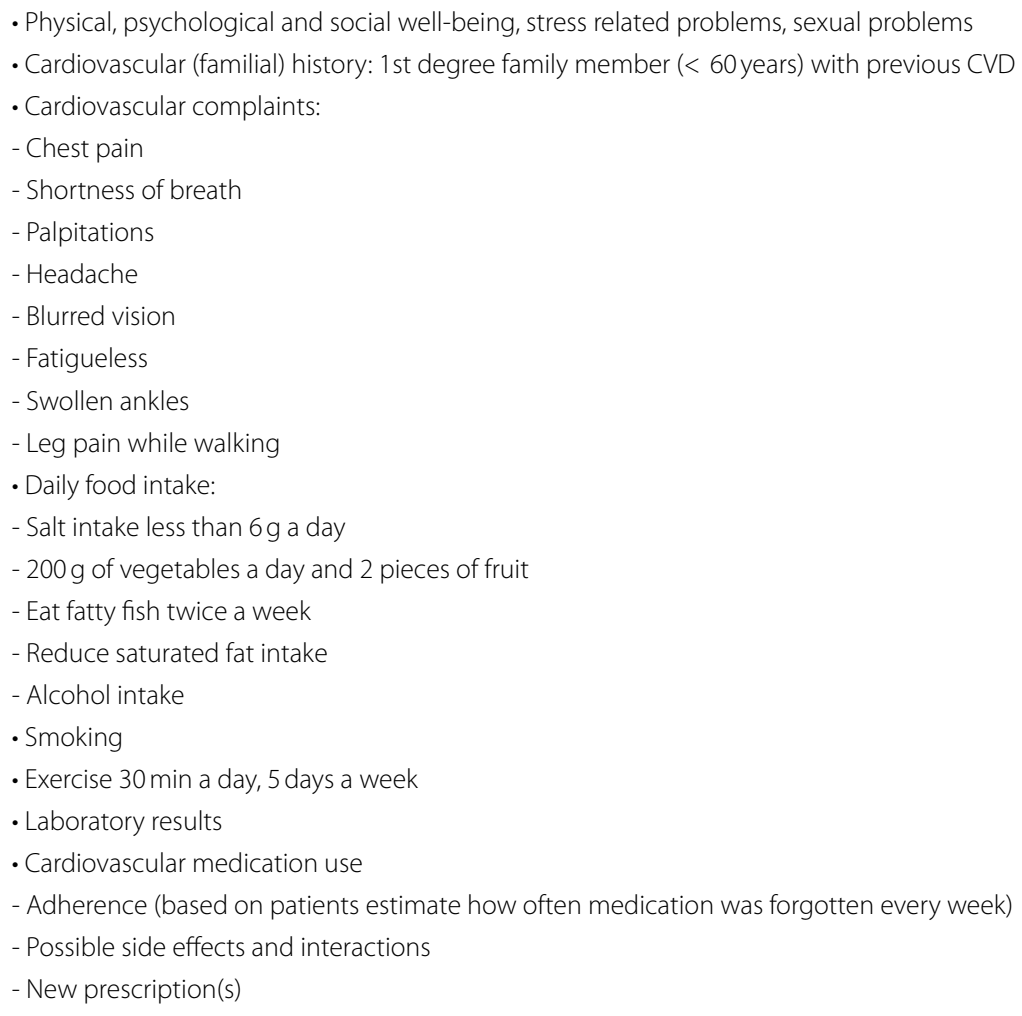

and pharmacological treatment. Although treatment goals were set in shared decision with the patient, in most cases targets values were used according to the 2006 CVRM guidelines of the Dutch Society of General Practice (4), which are listed in Supplementary Table 2.

If necessary, the PN referred the patient to a smoking cessation program, a physiotherapist or a dietician. If lifestyle advise resulted in insufficient effects on the risk factors level, blood pressure or lipid lowering medication could be initiated after consultation with the GP. When a cardiovascular event occurred or a patient failed to meet target values for blood pressure or lipids after maximum drug therapy, the GP referred to or consulted a specialist according to the criteria outlined in Table 3.

Regular follow up was agreed with the patient, depending on presence of comorbidity, complexity, achievement of treatment goals and advice according to the CVRM guideline. Patients were seen at least once a year by the GP to discuss laboratory results and seen 1-3 times a year by the PN to discuss lifestyle improvement and setting of new treatment goals if needed or wanted. In order to involve practices as much as possible in the development of the CVRM care program, the care group organized annual feedback meetings to discuss results and regular education for GPs and PNs on cardiovascularrelated subjects.

\section{Results}

Data collection and registration

In 2010 patient data (anamnestic, biometric, laboratory) were collected in a multidisciplinary registration system for integrated care (Care2U). Data registered in Care2U automatically ended up in the patients EHR and was accessible to the GP. Care2U automatically called patients for annual blood tests by linking the call to the date of birth. Due to registration problems in the EHR and to linking problems between 8 different EHR systems and Care2U, data collection was delayed and limited at the beginning. In the first quarter of 2013 these problems were finally solved. From April 2013, Care2U data were monitored monthly and every practice was able to view their data (process and outcome) any time.

\section{Patients participating in programmatic CVRM care}

On April 1st 2010, 30 practices (41 GPs) with 87,458 registered patients started with the selection process which led to the identification of 11,891 patients suitable for integrated CVRM care $(13,6 \%$ of the registered patients). Because 400 patients $(0,5 \%)$ eligible 
Table 3 Referral recommendations

Criteria for consultation of a nephrologist:

- Patients < 65 years with a eGFR 45-60 $\mathrm{ml} / \mathrm{min} / 1,73 \mathrm{~m} 2$

- Rapid deterioration of the renal function ( $>3 \mathrm{ml} / \mathrm{min} /$ year)

- Patients $>65$ years with a eGFR $30-45 \mathrm{ml} / \mathrm{min} / 1,73 \mathrm{~m} 2$

- Increase of albuminuria despite adequate pharmacological treatment

Criteria for referral to a specialist

- A new cardiovascular event

- Failure to meet target values despite adequate medication

- Familial dyslipidemia

- Premature, familial or undefined vascular disease

- Suspicion of secondary hypertension.

- Hypertension emerged in a short time and at young age (< 35 years)

- Suspected malignant hypertension (diastolic blood pressure $>120 \mathrm{mmHg}$ or clinical manifestations appropriate to cerebral complications like reduced consciousness, delirium, confusion, sudden impairment of vision or epileptic phenomena)

- Macro-albuminuria (Albumin-Creatinine Ratio $>30 \mathrm{mg} / \mathrm{mmol}$ ) and/or eGFR (<30 ml/min/1,73 m2)

- Patients with suspected underlying kidney disease, familial kidney disease or specific sediment abnormalities

for primary prevention, decided not to take part in the program and for 3037 patients $(3,5 \%)$ cardiovascular care was delivered in hospital or outpatient clinic, 8454 patients $(9,7 \%)$ participated in the CVRM care program: 5988 high risk patients $(6,8 \%)$ and $2466 \mathrm{CVD}$ patients $(2,8 \%)$. Due to quarterly renumeration, practices could start implementation every 3 months. In January 2013, 137 practices and 38,675 patients had started with programmatic CVRM care. Another 8 practices started with programmatic CVRM care in 2013, 2014 and 2015. Between 2016 and 20196 new practices affiliated to the care group. The number of practices and GPs, eligible patients and baseline characteristics of participating patients between 2010 and 2019 are listed in Table 4.

\section{Facilitating factors and barriers for implementation}

Facilitating factors for implementation were i) sufficient time given to PNs to provide patients with correct ICPC coding, ii) mandatory education for PNs and GPs on scrutinizing the EHR and iii) practice visitations by care groups' staff members every 3 months to discuss progress. Because all affiliated practices had given commitment, there were basically no serious barriers for

Table 4 Number of practices/GPs, eligibility and baseline characteristics of participating patients

\begin{tabular}{|c|c|c|c|c|c|c|c|c|c|c|}
\hline Year & 2010 & 2011 & 2012 & 2013 & 2014 & 2015 & 2016 & 2017 & 2018 & 2019 \\
\hline Practices $(n)^{a}$ & 42 & 122 & 137 & 140 & 143 & 145 & 148 & 149 & 151 & 151 \\
\hline GPS $(n)^{a}$ & 60 & 172 & 188 & 191 & 194 & 196 & 199 & 200 & 202 & 202 \\
\hline Registered pat ${ }^{a}$ & 87,458 & 378,099 & 402,623 & 406,119 & 416,433 & 422,296 & 401,077 & 405,956 & 422,452 & 436,009 \\
\hline Eligible pat ${ }^{a}$ & 11,896 & 50,937 & 54,990 & 61,709 & 63,846 & 65,692 & 64,877 & 65,166 & 67,194 & 68,989 \\
\hline$(\%)$ & 13,6 & 13,5 & 13,7 & 15,2 & 15,3 & 15,6 & 16,2 & 16,0 & 15,9 & 15,8 \\
\hline Particip pat ${ }^{b}$ & 8454 & 34,630 & 38,675 & 39,504 & 42,554 & 45,139 & 48,222 & 46,400 & 48,397 & 51,416 \\
\hline$(\%)$ & 9,7 & 9,2 & 9,6 & 9,7 & 10,2 & 10,7 & 12,0 & 11,4 & 11,5 & 11,8 \\
\hline Age (mean) $)^{b}$ & \pm & 67,2 & 67,8 & 68,1 & 68,5 & 68,9 & 69,5 & 69,5 & 69,8 & 70,1 \\
\hline Male $(\%)^{b}$ & \pm & 47,1 & 47,4 & 46,9 & 47,2 & 47,3 & 47,9 & 48,2 & 48,7 & 48.9 \\
\hline Second prev $(\%)^{b}$ & 29,2 & 28,6 & 33,3 & 35,3 & 37,0 & 38,2 & 40,6 & 43,9 & 44,3 & 45,2 \\
\hline$\geq 70$ Years (\%) & \pm & 42,6 & 43,6 & 44,7 & 46,2 & 47,6 & 50,5 & 51,5 & 52,8 & 54,3 \\
\hline
\end{tabular}

${ }^{a}$ Numbers from accountability reports (2010-2015) and quarterly reports (2016-2019)

${ }^{b}$ Numbers from Care2U data (2010-2019)

\pm Incomplete data

Second prev: Patients eligible for CVRM care with previous CVD 
implementation. Practicability in daily practice is mainly determined by sufficient time and care group support.

\section{Discussion}

\section{Summary}

This paper describes the implementation of a CVRM care program in 137 practices within less than 3 years for 38,675 patients, a number that increased to 51,416 patients in 2019, comprising around $11 \%$ of patient GP population. Facilitation factors were interest and commitment of GPs, sufficient allocated time for tasks, education linked to regular feedback and monitoring and a program based on approved guidelines.

\section{Comparison with existing literature}

The gap between evidence and real world practice has been identified long ago [9]. Yet, the evidence based on the best design and the best implementation strategies for prevention of cardiovascular disease is still limited. Already in 2010, Grol and co-workers, showed that commitment for chronic care, a stepwise and structured approach, sufficient staff support and implementation with regular feedback, are important facilitators [10]. This has recently been confirmed again by Wandell and co-workers that lack of time and renumeration were amongst the most important barriers for selective prevention of cardiovascular diseases [11]. Organizational changes in patient care, in terms of enhancing performance of non-physicians (practice nurses), computer systems integrated in the electronic health care records networks of the GPs, allow for automated reminders and clinical decision support have been known to be effective in improving patient care with reduced costs and improve patient outcomes with multidisciplinary teams and integrated care services [12]. Standard registration, feedback loops with training followed by improvement strategies and education as part of a learning health care system has been advocated. Our approach developed and applied in 2010 by deploying PNs and negotiating an adequate reimbursement, addressed most of these issue and perhaps therefore, experienced little barriers. In addition the our approach, a recent review from the SPIN-EU group came up with an evidence based generic "toolbox" for circumventing identified obstacles and harnessing facilitators in the design and implementation of cardiovascular prevention strategies. The development of the toolbox was based on data from five European countries, and may be of use in different communities, countries, and cultures [13].

\section{Strengths and limitations}

With all participating practices being affiliated to the care group, optimal reach, adoption and implementation of the CVRM program was assured. All practices in the Zuid-Oost Brabant region in the Netherlands were affiliated with the PoZoB care group. Practices covered rural, suburban and urban areas similar to the rest of the Netherlands, and can therefore be considered a representative sample of Dutch GPs.

The 2006 CVRM guidelines of the Dutch Society of General Practice has provided criteria to identify high risk patients [4]. When using structured files in the EHR as primary source for identification of eligible patients, the completeness and correctness relies on the ICPC and ATC coding of patients performed in general practice. The proportion of potentially eligible patients per practice based on ICPC and ATC codes varied between 14.8 and $30.3 \%$, suggesting that inadequate coding may exist, potentially leading to a considerable number of high risk patients who unfortunately remain out of sight. This is in line with an evaluation in 2012 of ICPC coding in 311 Dutch practices showing that ICPC coding varied substantially between practices and between EHRs [14]. We emphasized that adequate coding of disease and medication is an important prerequisite for implementation of a CVRM program in general practice. Another consideration is that most patients (men $\geq 55 \mathrm{y}$, women $\geq 60 \mathrm{y}$ ) with prescribed medication for hypertension and hypercholesterolemia were automatically included in the CVRM care program, while we did not know whether de diagnosis hypertension and hypercholesterolemia was set correctly according to the National CVRM Guideline [4], which may have led to overrepresentation. Monitoring the CVRM care program by means of quarterly reports ensures that results can be followed closely and adjusted if necessary. This is in line with the principles of the "Learning Healthcare System" (LHS) in which daily care data from electronic health records are compared and discussed to create continuous learning and improved health care delivery [15].

\section{Implications for research and/or practice}

Using a Multidisciplinary Information System for data collection gives the opportunity for monitoring (i) the development of the CVRM care program and (ii) performance of individual practices based on process and outcome indicators over a long period of time. With annual collection of biometric and laboratory data it is possible to assess improvements in cardiovascular risk factors and reduction in cardiovascular events. In feedback meetings GPs and PNs were able to discuss further development of the care program and in meetings between PNs and nurse staff experiences were exchanged. As such, evaluation follows a qualitative approach according to the RE-AIM framework, which is based on 5 elements (Reach, Effectivity, Adoption, 
Implementation, Maintenance) for assessing the impact of innovation on individual and organizational level $[16,17]$. The CVRM care program provides a solid basis for scientific evaluation on registration and outcomes of CVRM care and its determinants to explore practice variation and identify modifiable factors for improvement. Real-world data, which are becoming increasingly important in providing evidence of treatment effectiveness in clinical practice, allow us to evaluate the effect of our program in terms of improved cardiovascular outcomes and reduced cardiovascular events, and against which costs.

\section{Conclusion}

In conclusion, commitment of affiliated GPs, a structured approach with adequate coding of diagnoses and risk factors, central data registration and additional funding for sufficient staff support are important conditions for the introduction and implementation of successful and sustainable programmatic CVRM care. This approach constitutes the basis for long-term follow up and annual evaluation.

\begin{abstract}
Abbreviations
PoZoB: Praktijkondersteuning Zuidoost Brabant; CVRM: Cardiovascular risk management; GP: General Practitioner; ICPC: International Classification of primary Care; ATC-codes: Anatomical Therapeutic Chemical-codes; CVD: Cardiovascular diseases; DM: Diabetes mellitus; COPD: Chronic Obstructive Pulmonary Disease; PN: Practice Nurse; EHR: Electronic Health Record; CIS: Chain Information System; SCORE: Systematic Coronary Risk Evaluation; LHS: Learning Healthcare System; REAIM: Reach Effectivity Adoption Implementation Maintenance.
\end{abstract}

\section{Supplementary Information}

The online version contains supplementary material available at https://doi. org/10.1186/s12875-021-01602-w.

Additional file 1: Supplementary File 1. ICPC and ATC codes used to identify individuals potentially eligible for entering the CVRM care program.

Additional file 2: Supplementary File 2. Guidelines for treatment of patients enrolled in the CVRM care program based on 2006 CVRM guidelines of the Dutch Society of General Practice.

\section{Acknowledgements}

The contributing members of the PoZoB care group are listed alphabetically: Romeijnders A, MD, GP, medical director of PoZoB.

Rozema H, Manager care program Cardio Vascular Risk Management.

Wijnands C, Msc, epidemiologist.

\section{Authors' contributions}

The PoZoB Care Group designed the implementation protocol. GS drafted the first version of the manuscript. MH, SD and MB critically reviewed and revised the manuscript before providing final approval.

\section{Funding}

This research received no specific grant from any funding agency in the public, commercial or non-profit sectors.
Availability of data and materials

No patient data have been used for this paper.

\section{Declarations}

\section{Ethics approval and consent to participate}

Not applicable. All methods including the care groups protocol for data extraction, identification, examination and follow up were based on the 2006 CVRM guidelines of the Dutch Society of General Practice..

\section{Consent for publication}

Not applicable.

\section{Competing interests}

The authors declare that they have no competing interests.

\section{Author details}

${ }^{1}$ Bolwerk 11-14 5509, MH, Veldhoven, The Netherlands. ${ }^{2}$ Julius Center for Health Sciences and Primary Care, University Medical Center Utrecht, Heidelberglaan, 100 3584, CX, Utrecht, The Netherlands.

Received: 11 January 2021 Accepted: 3 December 2021

Published online: 05 January 2022

\section{References}

1. Global Health Estimates 2016. Deaths by Cause, Age, Sex, by Country and by Region, 2000-2016. Geneva: World Health Organization; 2018.

2. GBD 2013 DALYs and HALE Collaborators. Global, regional, and national disability-adjusted life years (DALYs) for 306 diseases and injuries and healthy life expectancy (HALE) for 188 countries, 1990-2013: quantifying the epidemiological transition. Lancet. 2015;386(10009):2145-91. https:// doi.org/10.1016/S0140-6736(15)61340-X Epub 2015 Aug 28. PubMed PMID: 26321261; PubMed Central PMCID: PMC4673910.

3. Yusuf $\mathrm{S}$, et al. Effect of potentially modifiable risk factors associated with myocardial infarction in 52 countries (the INTERHEART study): a case control study. Lancet. 2004;364:937-52.

4. Smulders YM, Burgers JS, Scheltens T, van Hout BA, Wiersma T. Simoons $\mathrm{ML}$; guideline development group for the Dutch guideline for multidisciplinary cardiovascular risk management. Clinical practice guideline for cardiovascular risk management in the Netherlands. Neth J Med. 2008;66(4):169-74 Review. PubMed PMID: 18424866.

5. Banga JD, Van Dijk JL, Van Dis I, Giepmans L, Goudswaard AN, Grobbee DE. NHG Standaard Cardiovasculair risicomanagement (eerste herziening). Huisarts Wet. 2012;55(1):14-28.

6. Nederlands Huisartsen Genootschap. NHG-standaard Cardiovasculair risico management ( tweede herziening). Huisarts Wet. 2019;62(4):55-7

7. Piepoli MF, Hoes AW, Agewall S, et al. 2016 European guidelines on cardiovascular disease prevention in clinical practice. The sixt joint task force of the European Society of Cardiology and Other Societies on Cardiovascular Disease Prevention in Clinical Practice ( constituted by representatives of 10 societies and by invited experts) Developed with the special contribution of the European Association for Cardiovascular Prevention \& Rehabilitation (EAPCR). Eur Heart J. 2016;37(29):2315-81.

8. $\vee$ Til JT, de Wildt JE, Struis JN: De organisatie van zorggroepen anno 2010. Huidige stand van zaken en de ontwikkelingen in de afgelopen jaren. RIVM rapport 260332001/2010 wwW.

9. Grol R, Grimshaw J. From best evidence to best practice: effective implementation of change in patient care. Lancet. 2003;362:1225-30.

10. Grol R, Braspenning J, Dijkstra R, Hulscher M, Wensing M. Implementation of NHG guidelines: success or problem? Huisarts Wet. 2010;53(1):42-6.

11. Wandell PE, de Waard A-KM, Holzmann MJ, et al. Barriers and facilitators among health professionals in primary care to prevention of cardiometabolic disease: a systematic review. Fam Pract. 2018;35(4):383-98.

12. Wensing M, Wollersheim $\mathrm{H}$, Grol R. Organizational interventions to implement improvements in patient care: a structured review of reviews. Implement Sci. 2006;1:2. https://doi.org/10.1186/1748-5908-1-2.

13. Sonderlund AL, Thilsing T, Korevaar J, Hollander M, Lionis C, Schellevis F, et al. An evidence-based toolbox for the design and implementation of 
selective-prevention primary-care initiatives targeting cardio-metabolic disease. Prev Med Rep. 2019;16:100979. https://doi.org/10.1016/j.pmedr. 2019.100979 PMID: 31508298; PMCID: PMC6722397.

14. Visser S, ten Veen P, Verheij R. Kwaliteit van ICPC-codering. Huisarts Wet. 2012;55(10):459.

15. Maddox TM, et al. The Learning Healthcare System and Cardiovascular Care. A scientific statement from the American Heart Association. Circulation. 2017;135:e826-57. https://doi.org/10.1161/CIR.0000000000000480.

16. Holtrop JS, Rabin BA, Glasgow RE. Qualitative approaches to the use of the RE-AIM framework: rationale and methods. BMC Health Serv Res. 2018;18:177. https://doi.org/10.1186/s12913-018-2938-8.

17. Gaglio B, Shoup JA, Glasgow RE. The RE-AIM framework: a systematic review of use over time. Am J Public Health. 2013;103:e38-46. https://doi. org/10.2105/AJPH.2013.301299.

\section{Publisher's Note}

Springer Nature remains neutral with regard to jurisdictional claims in published maps and institutional affiliations.

- fast, convenient online submission

- thorough peer review by experienced researchers in your field

- rapid publication on acceptance

- support for research data, including large and complex data types

- gold Open Access which fosters wider collaboration and increased citations

- maximum visibility for your research: over $100 \mathrm{M}$ website views per year

At BMC, research is always in progress.

Learn more biomedcentral.com/submissions 